\title{
Estudo do uso do eletrobisturi monopolar na hemostasia do complexo arteriovenoso ovariano de gatas submetidas à ovariohisterectomia eletiva
}

\author{
[Study of the use monopolar eletrobisturi in arteriovenous ovarian complex hemostasis of cats \\ undergone elective ovariohysterectomy]
}

\section{"Artigo Científico/Scientific Article"}

\author{
Thuani Camila Voltareli*, Leticia Iara Guizelini Bovo, João Vitor Veronez, Leila Isono Pereira, \\ Daniella Aparecida Godoi Kemper, Bernardo Kemper
}

Universidade Norte do Paraná, Arapongas-PR, Brasil.

*Autor para correspondência/Corresponding author: E-mail: voltarelithuani@gmail.com

\begin{abstract}
Resumo
Diferentes técnicas para hemostasia e secção do pedículo ovariano durante ovariohisterectomia $(\mathrm{OH})$ são relatadas na literatura. $\mathrm{O}$ uso da diatermia monopolar é um método de eletrocoagulação que viabiliza a estase vascular do ovário com eficiência. O presente trabalho objetivou avaliar a funcionalidade hemostática do Complexo Arteriovenoso Ovariano (CAVO) de felinos, com o uso da eletrocirurgia monopolar, sob a influência do peso dos animais em relação à incisão e período cirúrgico, quando comparados ao uso da técnica convencional. Vinte gatas hígidas, com peso entre 2,1 e $3,7 \mathrm{~kg}$, com idades variadas, foram submetidas à $\mathrm{OH}$ eletiva, onde, 10 gatas sofreram hemostasia e secção do CAVO sob uso da técnica das três pinças e 10 gatas mediante uso do eletrobisturi monopolar, de modo que o tempo cirúrgico foi quantificado sob uso de cronômetro e paquímetro era empregado na mensuração do tamanho da incisão. Analisando estatisticamente o presente experimento observou-se que o uso do eletrobisturi monopolar confere menor tempo cirúrgico total e tamanho de incisão, juntamente com boa eficácia hemostática do CAVO. No entanto, a variável peso dos animais não influenciou no tamanho da incisão e no tempo cirúrgico total.
\end{abstract}

Palavras-chave: castração; felinos; eletrocirurgia; diatermia; coagulação.

\begin{abstract}
Different techniques for hemostasis and ovarian pedicle section during ovariohysterectomy $(\mathrm{OH})$ are reported in the literature. The monopolar diathermy an electrocoagulation method that efficiently enables vascular stasis of the ovary. The present study aimed to evaluate the hemostatic functionality of the Feline Arteriovenous Complex (CAVO), using monopolar electrosurgery, under the influence of the animals' weight in relation to the incision and surgical period, when compared to the conventional technique. Twenty healthy cats, weighing between 2.1 and $3.7 \mathrm{~kg}$, with varying ages, underwent elective $\mathrm{OH}$, where 10 cats underwent hemostasis and section of the CAVO using the three forceps technique and 10 cats using the electroscope monopolar, so that the surgical time was quantified using a stopwatch and pachymeter was used to measure the size of the incision. Statistically analyzing the present experiment, it was observed that the use of the monopolar electrosurgical unit gives a shorter total surgical time and incision size, together with good hemostatic effectiveness of the CAVO. However, the variable animal weight did not influence the size of the incision and the total surgical time.
\end{abstract}

Keywords: castration; felines; electrosurgery; diathermy; coagulation.

\section{Introdução}

A ovariohisterectomia $(\mathrm{OH})$ é considerada um procedimento cirúrgico seguro e rotineiro quando efetivada em animais sadios (Lima et al., 2010). É frequentemente realizada não apenas com o intuito de controlar a superpopulação de 
cães e gatos, impossibilitando permanentemente a reprodução e em consequência reduzindo o abandono, os maus tratos e a população de animais errantes, mas também tendo em vista a propagação de zoonoses mediante a saúde pública (Lima et al., 2010; Conceição et al., 2016). A OH também tem influência perante a prevenção e tratamento de enfermidades ligadas à reprodução (Silveira et al., 2015).

Ackermann et al. (2014) citam que por tratar-se de animais com procriação excessiva, os métodos contraceptivos de felinos não devem ser enfatizados em machos, pois será efetivo apenas se $95 \%$ deles forem submetidos a tratamento. A cerca disso, diversos estudos sobre castrações em fêmeas vem sendo desenvolvidos, visando minimizar lesões cirúrgicas, apresentar custos viáveis e potencializar tempo de cirurgia (Costa Neto et al., 2009), posto que complicações intraoperatórias, pós-operatórias imediatas, mediatas ou tardias de $\mathrm{OH}$ são previstas (Santos et al., 2009).

Hemorragias são habitualmente a maior complicação transoperatória (Santos et al., 2009) e o fator mais comum de óbito após $\mathrm{OH}$, prevalentemente, resultantes do estiramento do ligamento suspensor ovariano, ligadura imprecisa dos pedículos do ovário ou através da secção do plexo e parede uterina (Santos et al., 2009; Tudury et al., 2014). Além disso, a hemorragia pode agravar-se caso a intervenção cirúrgica seja efetivada durante o estro, devido ao aumento da vascularização e intumescimento do trato genital (Santos et al., 2009; Watts, 2018).

Técnicas hemostáticas durante procedimentos cirúrgicos controlam e reduzem perdas sanguíneas. Costa Neto et al. (2018) apontam duas classes para a hemostasia: temporária, efetivada com pinças apenas ao longo do ato operatório, e definitiva, empregando diversos meios e instrumentos para obliteração do Complexo Arteriovenoso Ovariano (CAVO) e do útero: clipes vasculares, ligaduras ou transfixações com variados fios, braçadeiras de náilon (Silveira et al., 2015), diatermia monopolar e bipolar, cauterização por argônio, dispositivos de selagem de vasos (Watts, 2018) e laser de dióxido de carbono (MacPhail, 2014).

Referindo à hemostasia, Tudury et al. (2014) e Costa Neto et al. (2018) relataram o uso da eletrocirurgia bipolar em gatas submetidas à $\mathrm{OH}$ e concluíram que ao empregar a diatermia bipolar houve redução no tempo cirúrgico e hemostasia inalterada quanto às técnicas convencionais.

$\mathrm{Na}$ eletrocirurgia, a dinâmica ordenada de cargas elétricas é propagada ao designado tecido ao longo de um eletrodo ativo, que em atrito com a estagnação do tecido é alterada em calor, a qual regride ao indutor por um eletrodo neutro. $\mathrm{O}$ sistema monopolar difere-se do bipolar, pois os eletrodos apresentam-se distantes entre si, aumentando assim a densidade da corrente elétrica. Em cirurgias abertas, o fluxo da corrente não é influenciável negativamente, visto que o cirurgião tem uma ampla visualização do dispositivo (Trindade et al., 1998).

Devido à facilidade de acesso, desvantagem de custo e partindo da sapiência ao dispositivo, o presente estudo objetivou avaliar comparativamente o emprego do eletrobisturi monopolar na hemostasia do complexo arteriovenoso ovariano de gatas submetidas à ovariohisterectomia eletiva, via celiotomia mediana, através das variáveis: tempo cirúrgico, tamanho de incisão e ocorrência de hemorragia transoperatória, com o uso da técnica convencional de ligadura utilizando fio de Náilon através da técnica das pinças.

\section{Material e Métodos}

Foram usadas 20 gatas, hígidas, sem raça definida, com idade entre cinco meses e cinco anos, peso médio de 3,15 quilogramas $(\mathrm{kg})(2,1 \mathrm{a}$ $3,7 \mathrm{~kg}$ ), as quais foram submetidas à ovariohisterectomia eletiva.

Os pacientes foram submetidos a exame clínico geral, perfil hematológico, função renal e hepática para assegurar a higidez e registrar dados relativos à vida reprodutiva: número de cios, gestações e parto, para melhor divisão dos animais nos grupos correspondentes.

Os animais foram subdivididos aleatoriamente em dois grupos, os quais 10 animais (50\%) tiveram a hemostasia e secção do CAVO realizadas através de ligadura pela técnica das três pinças (grupo 1) e 10 animais (50\%) foram submetidos à hemostasia do CAVO através do uso do eletrobisturi monopolar (grupo 2). As gatas do grupo 1 obtiveram média de peso de 2,99 $\mathrm{kg}$, enquanto o grupo 2 atingiu peso médio de $3,11 \mathrm{~kg}$.

Os pacientes foram submetidos a exame clínico geral, perfil hematológico, função renal e hepática para assegurar a higidez e registrar dados 
relativos à vida reprodutiva: número de cios, gestações e parto.

O pré-operatório constituiu-se de jejum alimentar de até 12 horas e quatro horas de jejum hídrico (Castro et al., 2013). Os animais receberam analgesia pré-operatória, não esboçando sinais de dor no transoperatório, nem nas seis horas seguintes de observação pósoperatória. Medicações pré-anestésicas incluíram dexmedetomidina a $5 \mathrm{mg} / \mathrm{ml}$, na dosagem de 0,02 $\mathrm{mg} / \mathrm{kg}$ e morfina a $10 \mathrm{mg} / \mathrm{ml}$, na dose de 0,5 $\mathrm{mg} / \mathrm{kg}$. Tais medicações foram associadas em uma seringa e administradas por via intramuscular. Os pacientes foram induzidos com propofol a 10 $\mathrm{mg} / \mathrm{ml}$, na dosagem de $5 \mathrm{mg} / \mathrm{kg}$ e o plano anestésico foi mantido com isofluorano vaporizado com oxigênio em sistema semifechado. Empregou-se terapia antimicrobiana profilática com cefazolina na dose de $30 \mathrm{mg} / \mathrm{kg}$ por via endovenosa anterior à indução. Cloridrato de atipamezole na dosagem de $0,01 \mathrm{mg} / \mathrm{kg}$ foi administrado por via intramuscular no término da $\mathrm{OH}$. Na recuperação anestésica foram medicados com: dexametasona na dose de $0,25 \mathrm{mg} / \mathrm{kg}$ e cloridrato de tramadol na dosagem de $2 \mathrm{mg} / \mathrm{kg}$, ambos por via subcutânea.

$\mathrm{O}$ procedimento cirúrgico foi realizado em todas as etapas pelo mesmo cirurgião $\mathrm{e}$ posicionou-se a totalidade dos pacientes em decúbito dorsal e o abdome ventral, da cartilagem xifoide até o púbis, foi preparado assepticamente.

Em ambos os grupos experimentais a incisão foi realizada no terço médio do abdome caudal, iniciando três centímetros $(\mathrm{cm})$ caudal ao umbigo e estendida em direção ao púbis. Nas duas técnicas, o corno uterino foi localizado com o dedo indicador e tracionado para sua exteriorização (Figura 1. A).

No decorrer da técnica convencional empregando o uso de ligaduras para hemostasia do CAVO sob a técnica das três pinças, executouse uma fenda no mesovário, caudal ao CAVO, pela qual, duas pinças hemostáticas, tipo Crile reta $14 \mathrm{~cm}$ de comprimento, foram posicionadas para preensão do pedículo ovariano. Com o intuito de dificultar regressão venosa, em posição superior ao ovário colocou-se uma pinça hemostática, tipo Crile reta $14 \mathrm{~cm}$ de comprimento. Com uma tesoura, tipo Metzenbaum reta $14 \mathrm{~cm}$ de comprimento, o pedículo ovariano foi seccionado entre as duas pinças mais proximais. Com um fio, tipo Náilon 2.0, justapõe-se uma ligadura ao redor do CAVO e abaixo da pinça mais distal, a qual foi removida concomitante ao atar do nó. Uma pinça, do tipo dente de rato $14 \mathrm{~cm}$ de comprimento, foi usada para suster o pedículo ovariano e permitir que a pinça Crile remanescente fosse desvinculada. Conferiram-se sangramentos oriundos do CAVO e sendo inexistente, reposicionava-se o pedículo na cavidade abdominal. Similarmente prosseguiu-se a hemostasia e secção do CAVO contralateral.

A partir da exteriorização do corpo uterino e localização da cérvix, colocaram-se três pinças hemostáticas, tipo Crile $14 \mathrm{~cm}$ de comprimento, e seccionou-se o corpo do útero entre as duas pinças mais proximais, com uma tesoura, tipo Metzenbaum reta $14 \mathrm{~cm}$ de comprimento. Distal à terceira pinça, colocou-se uma ligadura transfixada em forma de oito, com fio tipo Náilon 2.0 , onde a pinça distal foi removida antes do aperto do primeiro nó. Uma pinça, do tipo dente de rato $14 \mathrm{~cm}$ de comprimento, foi usada para segurar o coto uterino durante a certificação quanto a hemorragias. Se ausente de sangramentos, o corpo uterino era reposicionado na cavidade abdominal.

Realizou-se a celiorrafia em três camadas: síntese da musculatura em padrão Sultan; síntese de subcutâneo em padrão ziguezague e; dermorrafia em padrão Sultan. Fio tipo Náilon foi utilizado na totalidade das suturas. Utilizando um paquímetro, mediu-se em milímetros $(\mathrm{mm})$ o tamanho da incisão.

Durante a técnica de hemostasia do CAVO sob uso do eletrobisturi monopolar, o mesovário sofreu uma abertura, caudal ao CAVO, pela qual uma pinça hemostática, tipo Crile curva $14 \mathrm{~cm}$ de comprimento, foi colocada para pinçar o pedículo ovariano. Acima do ovário, outra pinça hemostática, tipo Crile reta $14 \mathrm{~cm}$ de comprimento, foi posicionada a fim de evitar retorno venoso. Após isolamento do corno uterino e ovário em questão, a hemostasia do CAVO foi realizada por meio da diatermia monopolar com um eletrodo, tipo bola $4,2 \mathrm{~mm}$ de diâmetro, em uma pinça autoclavável do eletrobisturi (Bisturi Cirúrgico Eletrônico Monopolar 150 Watts BP 100 Plus Emai), em potência oito, com coagulação mista (corte e hemostasia). O eletrodo foi sobreposto à primeira pinça hemostática Crile, a qual conduzia a diatermia ao CAVO e completava a hemostasia (Figura 1. B). A secção do pedículo do ovário foi estabelecida através do uso de uma tesoura, tipo Metzenbaum reta $14 \mathrm{~cm}$ de comprimento, realizado acima da pinça Crile e 
abaixo do ovário (Figura 1. C). Com uma pinça, do tipo dente de rato $14 \mathrm{~cm}$ de comprimento, segurou-se o pedículo ovariano e então a pinça Crile remanescente foi liberada (Figura 1. D). Inspecionou-se sangramento do pedículo e este sendo ausente, o coto foi recolocado cuidadosamente na cavidade abdominal. Procedeu-se igualmente a hemostasia e secção no CAVO contralateral.

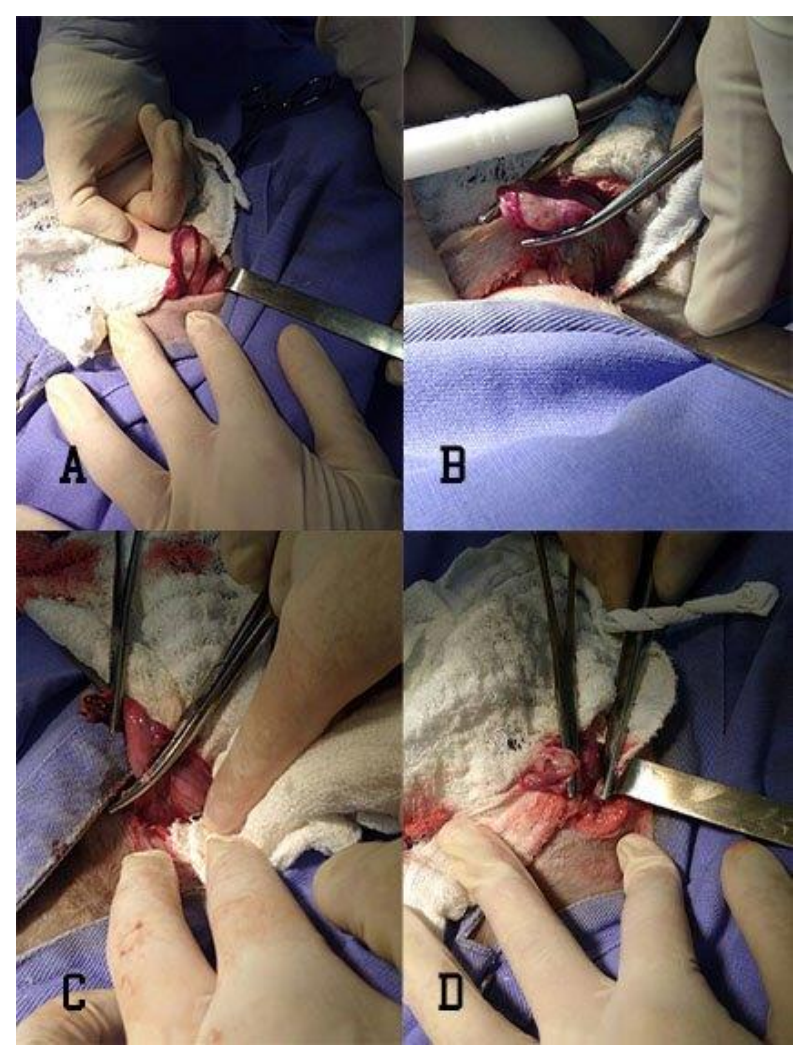

Figura 1. A. Exteriorização do ovário e inspeção do Complexo Arteriovenoso Ovariano (CAVO); B. Hemostasia do CAVO por meio da diatermia monopolar; C. Pedículo ovariano seccionado acima da primeira pinça Crile e abaixo do ovário; D. Inspeção de sangramento através do uso de uma pinça dente de rato.

A partir da exteriorização do corpo do útero e localização da cérvix, a metodologia cirúrgica foi equivalente à usada no grupo da técnica convencional.

Os procedimentos cirúrgicos da totalidade dos pacientes foram medidos quanto ao tempo cirúrgico integral: desde incisão até finalização da dermorrafia, com uso de cronômetro.

Perdas sanguíneas transoperatórias foram mensuradas referindo o contato visual e o uso de compressas cirúrgicas no decurso da análise hemorrágica de ambos CAVO's e vasos uterinos. Antes da rafia abdominal, uma compressa cirúrgica era posicionada no abdome do paciente; a qual era avaliada quanto à presença de fluidos sanguíneos.

Após a intervenção cirúrgica, os animais foram mantidos em observação por seis horas até receberem alta hospitalar. No pós-operatório receberam como método de restrição o uso de roupa cirúrgica ou de colar protetor e para uso oral prescreveu-se dipirona gotas, na dose de 25 $\mathrm{mg} / \mathrm{kg}$, a cada 24 horas, por três dias consecutivos, associado ao uso tópico de digluconato de clorexidina $1 \%$, a cada 24 horas, por dez dias. Retorno para avaliação clínica e retirada de pontos foi feita ao décimo dia de pósoperatório.

Após coleta de dados, foi realizada análise descritiva através de métodos tabelados e/ou mediante gráficos para variáveis quantitativas (mínimo, máximo, média, coeficiente de variação e desvio padrão). Igualmente realizou-se a teoria da análise de correlação linear com a finalidade de avaliar a significância da variável peso em relação ao tempo cirúrgico total e tamanho de incisão.

\section{Resultados e Discussão}

Realizou-se ovariohisterectomia eletiva em um total de 20 animais ( $\mathrm{n}=20)$, no qual 10 animais foram submetidos à ligadura do CAVO através da técnica das três pinças (grupo 1), onde o peso médio das gatas foi de $2,99 \mathrm{~kg}(\sigma 0,49 \mathrm{~kg})$ e o grupo experimental sob uso do eletrobisturi monopolar (grupo 2) foi composto de 10 gatas com peso médio de $3,11 \mathrm{~kg}(\sigma 0,45 \mathrm{~kg})$.

A cronometragem do tempo cirúrgico total, desde incisão até a dermorrafia, foi realizada de modo a avaliar sua restrição e consequente redução do período anestésico. Os resultados são expostos na Tabela 1, onde o grupo 2 resultou em uma média transoperatória de 948 segundos (15 minutos e oito segundos) evidenciando redução em relação ao período total de cirurgia através da técnica hemostática por meio do uso do eletrobisturi monopolar quando comparada aos resultados obtidos na realização da ovariohisterectomia eletiva em gatas por meio da técnica convencional, com três pinças (grupo 1), que demonstrou uma média de 1314 segundos (21 minutos e 9 segundos) com a mesma variável.

Watts (2018) em seu estudo com castração de caninos machos e fêmeas através do uso da diatermia bipolar relatou redução do tempo cirúrgico de 582 segundos (nove minutos e sete segundos) quando comparado com a técnica de 
ligadura com sutura, durante $\mathrm{OH}$ em cadelas empregando o eletrobisturi bipolar na hemostasia dos vasos do pedículo ovariano, então, concluiu que além da redução no tempo cirúrgico, o uso da diatermia foi eficiente e seguro. Costa Neto et al. (2018) ratificam em seus estudos a excelência e a facilidade de execução da técnica hemostática ademais à restrição do período cirúrgico total perante o uso do eletrobisturi bipolar em ovariohisterectomia de gatas ao defrontar à técnica de hemostasia prévia com ligaduras.

Em relação ao tamanho da incisão, o presente estudo obteve uma média de $18,10 \mathrm{~mm}$ de comprimento no grupo de gatas submetidas ao uso do eletrobisturi monopolar e média de 20,60 $\mathrm{mm}$ de comprimento no grupo proveniente à técnica convencional, valores expressos pela Tabela 2, em que dispôs redução respectiva de $11,90 \mathrm{~mm}$ e $9,40 \mathrm{~mm}$ quando comparada aos resultados obtidos por Tudury et al. (2014) através da realização de ovariohisterectomia em gatas ao contrapor o uso do eletrobisturi bipolar e a técnica de ligadura convencional com três pinças. Desta forma, as incisões utilizadas nas duas técnicas experimentais do presente estudo podem ser consideradas minimamente invasivas (Tudury et al., 2014)

Tabela 1. Análise descritiva da variável: tempo cirúrgico desde incisão até dermorrafia com o uso do eletrobisturi monopolar ou da técnica convencional de ligadura com três pinças para hemostasia do Complexo Arteriovenoso Ovariano (CAVO) de felinos.

\begin{tabular}{cccccc}
\hline Variável & Mínimo & Máximo & Média & $\begin{array}{c}\text { Desvio } \\
\text { Padrão }\end{array}$ & $\begin{array}{c}\text { Coeficiente de } \\
\text { variação (\%) }\end{array}$ \\
\hline & & & & & \\
Tempo cirúrgico (Grupo 1) & 1200 & 1560 & 1314 & 107,52 & 0,08 \\
Tempo cirúrgico (Grupo 2) & 900 & 1140 & 948 & 92,95 & 0,10 \\
\hline
\end{tabular}

Tempo - em segundos.

Tabela 2. Análise descritiva da variável: tamanho de incisão com o uso do eletrobisturi monopolar ou da técnica convencional de ligadura com três pinças para hemostasia do Complexo Arteriovenoso Ovariano (CAVO) de felinos.

\begin{tabular}{cccccc}
\hline Variável & Mínimo & Máximo & Média & $\begin{array}{c}\text { Desvio } \\
\text { Padrão }\end{array}$ & Coeficiente de variação \\
\hline & & & & & \\
Tamanho de incisão (Grupo 1) & 17 & 24 & 20,60 & 1,90 & 0,09 \\
Tamanho de incisão (Grupo 2) & 15 & 23 & 18,10 & 2,51 & 0,14
\end{tabular}

Tamanho de incisão - em milímetros.

A análise de correlação linear não constatou que a variável peso está relacionada significativamente com o tamanho de incisão em milímetros e com o tempo cirúrgico total em segundos, contrapondo Watts (2018) que verificou em seu estudo que cadelas mais pesadas (de 30 a $59,9 \mathrm{~kg}$ ) usufruíam em maior tempo cirúrgico total durante $\mathrm{OH}$ com uso da diatermia bipolar para hemostasia.

No exposto estudo, ao defrontar o uso do eletrobisturi para hemostasia do CAVO de felinos com o uso de ligaduras, benefícios como redução do tempo cirúrgico e consequente limitação do ciclo anestésico podem ser comprovados através dos dados obtidos, por conseguinte, há redução de custos operacionais em longo prazo para técnicas cirúrgicas de contracepção, o qual tem a viabilidade de coadjuvar no controle de animais errantes e reduzir abandono. A privação de reação granulomatosa no local da ligadura devido à ausência de corpo estranho como fios de sutura ou grampos cirúrgicos são aparentes, igualmente à exiguidade de rejeição ao material cirúrgico utilizado em ligaduras, visto que a hemostasia do pedículo ovariano era concluída idealizando a descoloração dos vasos, transpondo a região visivelmente branca como demonstrado por MacPhail (2014).

Desta forma, no transoperatório dos 20 animais, não foi constatado nenhuma hemorragia, contradizendo estudo de Tudury et al. (2014), o qual observou hemorragia transoperatória em $7,5 \%$ dos casos cirúrgicos, simultaneamente, Watts (2018) constatou perda sanguínea durante uma orquiectomia eletiva, ambos autores 
utilizaram em seus procedimentos cirúrgicos o eletrobisturi bipolar.

\section{Conclusão}

A eficácia hemostática, ausência de hemorragia transoperatória, o breve tempo utilizado e a redução do tamanho na incisão para realização de ovariohisterectomia eletiva com o uso do eletrobisturi monopolar em comparação com a técnica convencional utilizando três pinças, conferem à técnica uma boa possibilidade, funcionalidade e exequibilidade cirúrgica, visando à facilidade de acesso ao eletrobisturi monopolar, redução de custos cirúrgicos, diminuição na possibilidade de reações locais ao fio de ligadura do CAVO, formação de granulomas e redução no tempo cirúrgico total, tendo em vista igualmente a redução no período e risco anestésico.

\section{Conflito de Interesse}

Os autores declaram não existir conflito de interesse.

\section{Comitê de Ética}

O projeto de pesquisa foi aprovado pelo comitê de ética da Universidade Norte do Paraná, sob o número 003/18.

\section{Agradecimentos}

Agradecemos à Universidade pela oportunidade da realização do estudo e aos tutores pela confiança e disponibilidade.

\section{Referências}

Ackermann, C.L.; Silva, T.F.P.; Silva, L.D.M.; Lopes, M.D. Métodos contraceptivos em gatas domésticas - Revisão de literatura. Ciência Animal, 24 (2): 41-54, 2014.

Castro, J.L.C.; Santalucia, S.; Castro, V.S.P.; Cancian, M.A.; Mejolaro, S.; Trindade, A.B.; Soares, A.V.; Raiser, A.G. Jejum préoperatório em cães e gatos - Revisão de literatura. Medvep - Revista Científica de Medicina Veterinária - Pequenos Animais e Animais de Estimação, 11(37): 22-637, 2013.

Conceição, M.E.B.A.M.; Teixeira, P.P.M.; Dias, L.G.G.G. Perspectivas acerca da esterilização cirúrgica em cadelas e gatas. Investigação, 15(1): 10-15, 2016.

Costa Neto, J.M.; Teixeira, E.M.; Ferreira Filho, E.M.; Toríbio, J.M.M.L.; Almeida Filho, C. H.R., Moraes, V.J. Braçadeiras de náilon para hemostasia

preventiva

na

ovariosalpingohisterectomia em gatas. Revista Brasileira de Saúde e Produção Animal, 10(3): 615-624, 2009.

Costa Neto, J.M.; Blanco, N.S.; Andrade, V.M.; Souza, T.D.C.; Borges, K.B.; Miranda, R.A.; Martins Filho, E. F.; Quesada, A. M. Uso da diatermia bipolar para obliteração vascular em ovariohisterectomia de gatas. Jornal Interdisciplinar de Biociências, 3(1): 5-10, 2018.

Lima, A.F.M.; Pardini, L.; Luna, S.P.L. Avaliação de sobrevida, alterações genitourinárias, comportamentais e de peso corpóreo no pósoperatório tardio em cadelas e gatas submetidas à ovariosalpingohisterectomia sob diferentes métodos de ligadura do pedículo ovariano. Ars Veterinaria, 26(2): 60-65, 2010.

MacPhail, C.M. Biomateriais, sutura e hemostasias. In: Fossum, T.W. Cirurgia de pequenos animais. $4^{\mathrm{a}}$ ed. Rio de Janeiro: Elsevier, 2014. p.74-87.

Santos, F.C.; Corrêa, T.P.; Rahal, S. C.; Crespilho, A.M.; Lopes, M.D.; Mamprim, M.J. Complicações da esterilização cirúrgica de fêmeas caninas e felinas. Revisão de literatura. Veterinária e Zootecnia, 16(1): 8-18, 2009.

Silveira, C.P.B.; Araújo, M.S.C.; Horr, M.; Alencar, E.A.M.; Rigaud, R.; Oriá, A.P.; Martins Filho, E.F.; Costa Neto, J.M. Validação de técnica hemostática do complexo arteriovenoso ovariano na ovariosalpingohisterectomia de gatas. Ciência Animal Brasileira, 16(1): 81-92, 2015.

Trindade, M.R.M., Grazziotin, R.U., Grazziotin, R.U. Eletrocirurgia: sistemas mono e bipolar em cirurgia videolaparoscópica. Acta Cirúrgica Brasileira, 13(3): 1998.

Tudury, E.A.; Almeida, A.C.M.; Tavares, T.H.T.F.; Fontes, K.M.H.A.S.; Borges, D.C. Hemostasia e incisão do pedículo ovariano de gatas submetidas à ovariossalpingohisterectomia eletiva por meio da técnica das três pinças ou do eletrobisturi bipolar. Estudo comparativo. Arquivo Brasileiro de Medicina Veterinária e Zootecnia, 66(5): 1427-1434, 2014.

Watts, J. The use of bipolar electrosurgical forceps for haemostasis in open surgical ovariectomy of bitches and queens and castration of dogs. Journal of Small Animal Practice, 59(8): 465-473, 2018. 